\title{
Low Cost Smart Stick for Blind and Partially Sighted People
}

\author{
Mithiles Kumar, Md Faysal Kabir and Sahadev Roy
}

\begin{abstract}
The paper represents a novel approach to overcome the major challenges face by blind and partially blind people to cross the road and read. The proposed voice stick used to cross the road and reach their destination without any support. It is introduced a new technique to read printed material using Android phone. Low cost able to serve as a smart blind stick being more efficient and helpful than the conventional one. This will assist the blind persons during the walk and provides an alarm if any hurdle is detected within the set range.
\end{abstract}

Keywords

Blind stick, large obstacle avoidances, Small Obstacle Avoidances

\section{INTRODUCTION}

Major difficulties faced by blind people to cross road or path finding without help of others [1]. This project helps them to facilitate in path clearing assistance and collision detection. We can able to incorporate voice stick [2] which also used to read the printed material which provides an audio output. If any obstacle as well as any type of hazard conditions are detected the corresponding alarm alert them. The proposed device works in both night and day and it is very reliable and effective. The proposed walking stick for the blind may be useful for control and monitoring using ad-hoc network where continuous safety is required. This will assist the blind persons during the walk and provides an alarm if any hurdle is detected within the set range.

The above shown Fig. 1 is an Intelligent Voice Stick with multiple input and output features as represented in it. There are five sensors namely ultrasonic, infrared, water, fire and light (LDR) which are used for sensing different conditions and depending upon which the processor will update its input and access the output devices as its response to the input. The system is having four different output devices i.e., speech instructor, vibrator, buzzer and flashlight which are accessed in different combinations depending upon different inputs state.

When a visually impaired person is walking in the street, he may encounter several problems in the way such as obstacles like human, animal or wall, pit or staircase, muddy surface, fire and many others which can create troubles like accident or injuries to him even after holding conventional sticks. If the person is holding this electronic intelligent voice stick while walking it will help him to protect himself from these hurdles. An easy path finding technology [3] may be used in this device. A low cost microcontroller [4] [5]is useful for this design.

\section{Proposed Design}

The design aim is to provide a low cost smart stick to the blind and partially sighted people. The system overview is shown in the Fig. 1. The arrangement of the five sensors Ultrasonic, Infrared, Water, Fire and Light (LDR) which are used in the stick, also shown in Fig. 1.

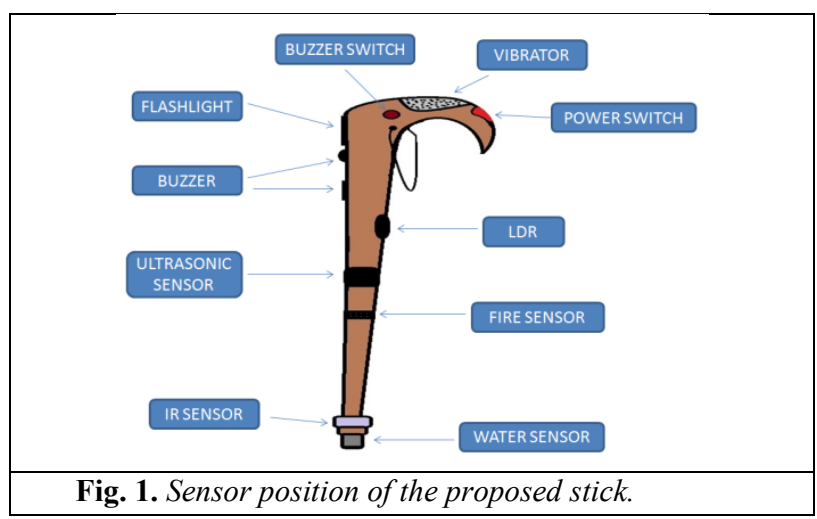

Operations of these five sensors are discussed briefly in the following subsections.

\section{A. Large Obstacle Detection Methodology}

While walking if any obstacle like human, animal, tree or wall appears in front of him then the Ultrasonic sensor [6] which is continuously transmitting high frequency sound waves are echoed back after striking the obstacle and are collected across receiver. These received waves are sending to the processor in the form of electrical impulse for processing [7]. After processing the input the processor will sent a voice instruction stored within the memory for obstacle avoidance and at the same time vibrator also shakes to alert the person regarding presence of obstacle and 
thus provide enough time to change his path for avoiding possibilities of collision.

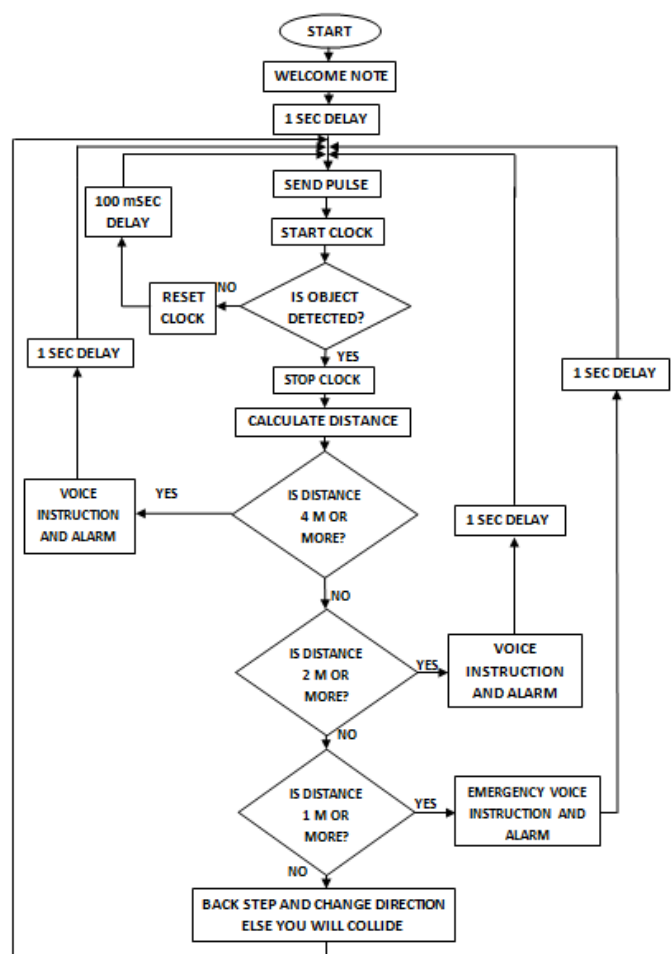

Fig. 2. Flowchart of the proposed embedded system

The flow chart (Fig. 2) of obstacle detector using ultrasonic sensor which is basically having two parts [8], first part deals with the obstacle detection while second part deals with distance measurement and alerting the user depending on distance of the obstacle to avoid collision. When the system is switch on, it starts with well wishing to the user. After a delay of one second the ultrasonic sensor transmits the high frequency wave of $40 \mathrm{kHz}$ to check the obstacle in front and at the same time it starts the timer to calculate the time elapsed, and waits for echo signal to receive at receiver within the time limit. If no signal received at receiver end then reset the timer and again sends the wave, this process is repeated until any object is not detected. If the echo waves are received at receiver end then the timer is stopped and the time taken to receive the transmitted signal after echo is used to calculate the object distance from user.

Depending on distance of the obstacle from the person four zones are formed i.e., far (safe) zone, near zone, close zone and danger zone. If the detected object is at 4 meter or more then it comes under far (safe) zone, the user will be informed through a voice instruction about presence of an obstacle at 4 meter distance. If the object is found at 2 meter or more then it comes under near zone, and a voice instruction along with vibrating alert signal will be send to the user so that he will be mentally ready and slow down his speed of walking and try to find the obstacle free path by directing sensor in different direction. If the object is found at 1 meter or more then it comes under close zone, an emergency voice instruction along with vibrating alert will be send to user to stop at the same place and to find the obstacle free direction using the sensor. And if object is detected at less than 1 meter then it comes under danger zone, a voice instruction to back step along with vibration alert is send to the user and at the same time an buzzer will also rung to let people around know that the person is blind and he needs help.

\section{B. Small Obstacle Detection Methodology}

If in the way pit, staircase or stone like obstacle which are small in size and cannot be detected by the Ultrasonic sensor [9] are detected by the IR sensor as it is located at the lower side of the stick. After detecting the small obstacles on ground, IR sensor will send the signal to the processor and as a result of which the processor will send a voice instruction for small obstacle available on ground from the memory location and at the same time it will enable the first buzzer for informing the blind person about presence of obstacle on ground and thus avoidance the collision.

\section{Muddy Surface Detection Methodology}

Now, if the surface is wet or muddy it can cause slipping on the floor and thus can hurt [10]. In order to have precaution against the wet surface a Water sensor is located at the base of stick. When the Water sensor comes in contact of the wet surface its resistance changes and produced electrical signal which trigger the processor [11]. Immediately the processor will send a voice instruction for wet surface and at the same time it will enable the second buzzer for informing about presence of wet or muddy floor.

\section{Fire Detection Methodology}

While walking there can be a situation that something is burning or igniting in the way. If the ignited body is radiating a lot of heat then the person can easily sense the heat with the help of his skin and can keep himself away. But if the heat is not sensed by the skin properly, it can lead to a dangerous accident [12]. In order to protect the person against this hazardous situation a heat sensor is introduced in the stick which is very sensitive to the heat and can detect the heat from long distance [13], also we can identify the direction from which it is radiating by moving the stick in different direction. If the sensor detects the heat radiation it will send a electrical signal to controller and thus voice instruction will be send to person and at the same time vibrator and first buzzer also start alarming. 


\section{E. Smart Night Lamp}

Walking in public place during night time is one of the challenging task for visually impaired peoples because others peoples will not notice that the person is blind and in rush they may accidentally clash with them. In order to overcome this problem, an LDR sensor is used in the Smart stick whose resistances change due to change of the light intensity [14]. During night the LDR will have high resistive (M ohm) path and no current pass through it but through a LED connected parallel with it. Due to this flow of large current the LED connected in front of the stick illuminates brightly and acts as a Flashlight which can be easily noticed by others. It alerts the people around about the presence of blind person and thus provides him sufficient space to pass the way.

\section{FUTURE WORK}

Till now we have succeeded to translate English language text into speech output of three different languages i.e., English, Hindi and Bengali which are the most commonly spoken and understand by the people of India. We are planning to include some other local languages so that several other visually impaired people can use this stick to overcome the reading problem in daily life. We are also working out to include Face recognition system that will avail blind people to recognize the person standing in front of him. At the same time global positioning system (GPS) can be linked with the voice stick for navigation, so that person can know his current position and distance from the destination which will be informed to user through voice instructions whenever needed.

\section{CONCLUSION}

This product is an attempt to fill the gap formed in visually impaired people's life by providing them strength of self dependency. Here we have designed an electronically smart guiding stick that is incorporated with multiple sensors which will help in navigating the way while walking and keep alarming the person if any sign of danger or inconvenience is detected. This stick also comprises of Text to speech conversion that facilitates the translation of English language text into speech output in different languages that will help blind and partially sighted people over worldwide to get audio news update from news paper, study books and hand written notes. This can also be used by the students for hearing the audio of class notes and understanding the meaning in compatible language. The system has been designed giving priority to safety, comfort, cost effectives and strength of the product. It can be further improved to increase its capability of decision making with introducing some other sensors in the system that will increase the area of application.

\section{REFERENCES}

[1] J. M. Loomis, R. G. Golledge and R. L. Klatzky, "Navigation System for The Blind: Auditory Display Modes and Guidance," Presence: Teleoperators and Virtual Environments, vol. 7, no. 2, pp. 193-203, 1998.

[2] V. Hanumante, R. Debnath, D. Bhattacharjee, D. Tripathi and S. i Roy, "English Text to Multilingual Speech Translator Using Android," International Journal of Inventive Engineering and Sciences, vol. 2, no. 5, pp. 4-9, 2014.

[3] V. Hanumante and S. M. S. Roy, "Low Cost Obstacle Avoidance Robot," International Journal of Soft Computing and Engineering, vol. 3, no. 4, pp. 52-55, 2013.

[4] R. Srivastava, N. Kumar, B. Anjali and S. N. Singh, "Grid Interactive Solar Powered Automated Bottling Plant Using Microcontroller," International Journal of Advanced Engineering and Management, vol. 2, no. 1, pp. 9-14, 2017.

[5] V. Sukhadeve and R. Sahadev, "Advance Agro Farm Design With Smart Farming, Irrigation And Rain Water Harvesting Using Internet of Things," International Journal of Advanced Engineering and Management, vol. 1, no. 1, pp. 33-45, 2016.

[6] J. Borenstein and Y. Koren, "Obstacle avoidance with ultrasonic sensors," IEEE Journal on Robotics and Automation, vol. 4, no. 2, pp. 213-218, 1988.

[7] B. Johann and Y. Koren, "Real-time obstacle avoidance for fast mobile robots," IEEE Transactions on systems, Man, and Cybernetics, vol. 19, no. 5, pp. 1179-1187, 1989.

[8] T. A. Sheikh, S. Dutta, S. Baruah and P. R. S. Sharma, "Obstacle Avoidace Robot Using LabView," IAES International Journal of Robotics and Automation, vol. 4, no. 3, pp. 164167 , 2015 http://dx.doi.org/10.11591/ijra.v4i3.978.

[9] J. Borenstein and Y. Koren, “ The vector Field Histogram-Fast Obstacle Avoidance for Mobile 
Robots," IEEE Transactions on Robotics and Automation, vol. 7, no. 3, pp. 278-288, 1991.

[10] P. Keeton, "Personal Injuries Resulting from Open and Obvious Conditions," University of Pennsylvania Law Review, vol. 100, no. 5, pp. 629-648, 1952.

[11] S. Lam Po Tang and G. K. Stylios, "An Overview of Smart Technologies for Clothing Design and Engineering.," International Journal of Clothing Science and Technology, vol. 18, no. 2, pp. 108-128, 2006.

[12] R. G. Millikan, "Biosemantics," The Journal of Philosophy, vol. 86, no. 6, pp. 281-297, 1989.

[13] R. Elghanian, J. J. Storhoff, R. C. Mucic, R. L. Letsinger and C. A. Mirkin, "Selective
Colorimetric Detection of Polynucleotides Based on the Distance-Dependent Optical Properties of Gold Nanoparticles," Science , vol. 277, no. 5329, pp. 1078-1081, 1997.

[14] Vipasha and P. Abrol, "Design of Traffic Flow based Street Light Control," International Journal of Computer Applications, vol. 72, no. 18, pp. 32-37, 2013.

\section{Mithiles Kumar}

Dept. of ECE, NIT Arunachal Pradesh, India

mithileshkumarrr@gmail.com

Md Faysal Kabir

juel.kabir@gmail.com

Dumkal Polytechnic, India

Sahadev Roy

Dept. of ECE, NIT Arunachal Pradesh, India sahadevroy@gmail.com 